\title{
Sensing Cold-Induced Situational Impairments in Mobile Interaction Using Battery Temperature
}

\author{
ZHANNA SARSENBAYEVA, The University of Melbourne \\ NIELS VAN BERKEL, The University of Melbourne \\ AKU VISURI, University of Oulu \\ SIRKKA RISSANEN, Finnish Institute of Occupational Health \\ HANNU RINTAMAKI, Finnish Institute of Occupational Health \\ VASSILIS KOSTAKOS, The University of Melbourne \\ JORGE GONCALVES, The University of Melbourne
}

\begin{abstract}
Previous work has highlighted the detrimental effect of cold ambience on fine-motor skills during interaction with mobile devices. In this work, we develop a method to infer changes in finger temperature of smartphone users without the need for specialised hardware. Specifically, we demonstrate that smartphone battery temperature is a reliable gauge for determining changes to finger temperature. In addition, we show that the behaviour of smartphone battery temperature in cold settings is consistent across different smartphone models and battery configurations. Our method can be used to determine coldinduced situational impairments, and trigger interface adaptations during mobile interaction.
\end{abstract}

CCS Concepts: • Human-centered computing $\rightarrow$ Empirical studies in HCI; • Human-centered computing $\rightarrow$ Ubiquitous and mobile computing; • Human-centered computing $\rightarrow$ Smartphones

\section{KEYWORDS}

Smartphones, ambient temperature, battery temperature, situational impairments, finger temperature, cold chamber.

This work is partially funded by the Academy of Finland (Grants 276786-AWARE, 286386-CPDSS, 285459-iSCIENCE, 304925-CARE), the European Commission (Grant, 6AIKA-A71143-AKAI), and Marie Skłodowska-Curie Actions (645706-GRAGE).

Author's addresses: Z. Sarsenbayeva, The University of Melbourne, School of Computing and Information Systems, Parkville 3010, Melbourne, Australia; email: zsarsenbayev@student.unimelb.edu.au; N. van Berkel, The University of Melbourne, School of Computing and Information Systems, Parkville 3010, Melbourne, Australia; email: n.vanberkel@student.unimelb.edu.au; A. Visuri, University of Oulu, Center for Ubiquitous Computing, Pentti Kaiteran katu 1, PO Box 4500, FI-90014 Oulu, Finland; email: aku.visuri@oulu.fi; S. Rissanen, Finnish Institute of Occupational Health, PL 40, 00251 Helsinki, Finland; email: sirkka.rissanen@ttl.fi; H. Rintamaki, Finnish Institute of Occupational Health, PL 40, 00251 Helsinki, Finland; email: hannu.rintamaki@ttl.fi; V. Kostakos, The University of Melbourne, School of Computing and Information Systems, Parkville 3010, Melbourne, Australia; email: vassilis.kostakos@unimelb.edu.au; J. Goncalves, The University of Melbourne, School of Computing and Information Systems, Parkville 3010, Melbourne, Australia; email: jorge.goncalves@unimelb.edu.au

Permission to make digital or hard copies of part or all of this work for personal or classroom use is granted without fee provided that copies are not made or distributed for profit or commercial advantage and that copies bear this notice and the full citation on the first page. Copyrights for components of this work owned by others than ACM must be honored. To copy otherwise, distribute, republish, or post, requires prior specific permission and/or a fee. Request permissions from permissions@acm.org. $2474-9567 / 2017 / 9-98 \$ 15.00$

Copyright is held by the owner/author(s). Publication rights licensed to ACM.

DOI: http://doi.org/10.1145/3130963 


\section{INTRODUCTION}

Cold ambience is known to affect fine-motor skills during interaction with mobile devices. This is due to the fine-motor dexterity loss caused by drops in finger temperature [6,13]. However, while previous work has shown that finger temperature is an important consideration when designing mobile phone applications, no reliable way of inferring finger temperature in situ has been proposed without the need for external measurement devices (e.g., skin thermistors).

Future smartphones may come with embedded environmental temperature sensor to gauge ambient temperature, but this is still not the case today. In our work, we make creative use of existing smartphone sensors to gauge finger temperature. Specifically, we investigate if finger temperature can be inferred from the smartphone's battery temperature. The literature contains a growing list of examples where smartphone sensors originally intended for a particular function can actually be appropriate for a wholly different use case. For example, researchers have shown how to transmit data using the magnetometer [9], or determine social context through Bluetooth traces [2].

Here we demonstrate that it is possible to leverage data on smartphone battery temperature to infer fluctuations of a user's finger temperature during interaction. We show a significant correlation between battery and finger temperature, and as such one can be used to infer the other. Our method can infer changes in users' finger temperature using off-the-shelf smartphones without the need for additional sensors or hardware.

\section{RELATED WORK}

\subsection{Environmental Sensing Using Smartphones}

Previous work has described the use of smartphone sensors to better understand surrounding weather conditions. For instance, Mass \& Madaus [10] discuss the use of smartphone pressure sensors for surface pressure observations. They describe that a fine-grained network of smartphone users would allow for highresolution weather prediction as pressure readings are "not influenced by characteristics of the underlying surface, as are temperature and wind" [10], and therefore readings are not influenced by different contexts (e.g., shade, urbanisation).

Overeem et al. [12] also suggest that smartphones can be used as an effective instrument to study environmental and climate changes. Sensor data such as air humidity, air pressure, and air temperature can be used for data assimilation in weather prediction models, water management, and urban planning [12]. More relevant to our work, they report the analysis of a six-month dataset of 2.1 million battery temperature readings from eight major cities collected using the OpenSignal ${ }^{1}$ Android application. They found that daily battery temperature was strongly correlated with the observed daily air temperature $(r=0.82)$ [12]. In addition, literature shows a substantial impact of ambient temperature on human's body temperature, particularly for extremities (e.g. fingers, toes) [11]. These findings provide us with strong motivation towards investigating the possibility of inferring changes in user finger temperature based on changes in smartphone battery temperature.

\subsection{Extending Smartphone's Capabilities}

The literature also contains examples on the use of smartphones in combination with specialised devices for environmental sensing. A combination of general smartphone sensors and purpose-made (add-on) sensors allows for the collection of sensor data not feasible with a standalone smartphone. By distributing 8000 small add-ons for regular smartphones among the Dutch population, Snik et al. [14] deployed a 'citizen science' experiment to carry out aerosol measurements of high temporal resolution. "The optical design of iSPEX uses the smartphone camera as the detector, and the iSPEX add-on produces a spectrum of the light that entered the slit [...]" [14].

\footnotetext{
${ }^{1}$ http://opensignal.com
} 
In a different project, researchers developed an attachment for smartphones to crowdsource the collection of pollution data [8]. Combining this data with the GPS sensor of the mobile phone allowed for a more detailed understanding of urban air pollution.

Aram et al. [1] monitored changes in temperature and humidity using a Bluetooth-based acquisition system. The system consisted of device with a built-in temperature and humidity sensor, and a microcontroller wirelessly transmitting the climatic parameters to a receiver via Bluetooth. The device was left in a climatic chamber to monitor the temperature drop from $25{ }^{\circ} \mathrm{C}$ down to $-20^{\circ} \mathrm{C}$ followed by a temperature rise back to 25 ${ }^{\circ} \mathrm{C}$, as well as a constant humidity level. The results show that temperature values obtained by the system were consistent with the temperature values inside the climatic chamber, but not for humidity values. They suggest that their approach might be useful to observe climate conditions for small environments, such as laboratories, home rooms, or medical spaces, and to trigger alarms when these conditions change [1].

\subsection{Impact of Situational Impairments on Smartphone Use}

Previous work suggests that smartphone interfaces should be adapted when used under various situational impairments, such as dynamic state of the phone [5], ambient light and noise [16], movement [4], and cold ambience [6,13]. A number of different solutions have been developed to overcome these situational impairments when interacting with a mobile device. For example, Goel et al. [4] suggest adapting the keyboard interface when walking is detected to improve typing experience. This adaptive interface reduced errors and increased typing speed. Moreover, Wobbrock [16] argues that a better understanding of situational impairments can in addition contribute to improved accessibility and adaptive user interfaces. Furthermore, he considers the possibility that solutions designed for people with physical impairments can be applied to those with situational impairments during mobile interaction (e.g., finger arthritis and people with cold fingers [16]).

On the topic of situational impairments caused by cold temperatures, previous work has shown that user performance when completing tapping tasks on a smartphone is affected by finger temperature. As a result of this finding, the researchers suggest adding finger temperature as a parameter in Fitts' Law modelling [6]. Furthermore, Sarsenbayeva et al. [13] show that precision and quickness are adversely affected by cold temperatures. While the researchers provide design suggestions for mobile device interface adaptation in cold environments, they do not describe how user exposure to cold ambience can be detected in a naturalistic setting (i.e., without temperature sensors attached to the user's fingers). Hence, we extend previous work by proposing a method to determine changes in finger temperature by considering the smartphones' battery temperature.

\section{STUDY}

We conducted two experiments to 1) assess the effect of a cold environment on the battery temperature of several smartphone models, and 2) investigate the relationship between smartphone battery temperature and human finger temperature.

\subsection{Experiment 1: Device Comparison}

We conducted a comparative study of changes in battery temperature over time using multiple handsets. Our objective was to investigate whether different phones and batteries behave similarly in cold settings. We considered four different smartphones that vary in manufacturer, size, battery capacity, and other factors (see Table 1). Given the wide range of characteristics in our selected phones, we argue that they provide a good representation of existing models in the market. 
Table 1. Specifications of smartphones used in our study.

\begin{tabular}{lllll} 
& $\begin{array}{l}\text { Motorola } \\
\text { Moto G3 }\end{array}$ & $\begin{array}{l}\text { Samsung } \\
\text { Galaxy S4 } \\
\text { Mini }\end{array}$ & $\begin{array}{l}\text { Huawei } \\
\text { Nexus 6P }\end{array}$ & $\begin{array}{l}\text { LG } \\
\text { Nexus 5X }\end{array}$ \\
\hline Dimensions & $142.1 \times 72.4 \times$ & $124.6 \times 61.3 \times$ & $159.3 \times 77.8 \times$ & $147.0 \times 72.6 \times 7.9$ \\
(H x D x W) & $11.6 \mathrm{~mm}$ & $8.49 \mathrm{~mm}$ & $7.3 \mathrm{~mm}$ & $\mathrm{~mm}$ \\
Weight & $155 \mathrm{~g}$ & $107 \mathrm{~g}$ & $178 \mathrm{~g}$ & $136 \mathrm{~g}$ \\
Screen size & $5.0 ”$ & $4.3 ”$ & 5.7 & $5.2 ”$ \\
Body material & Polycarbonate & Polycarbonate & Metal & Polycarbonate \\
Battery type & Li-Ion & Li-Ion & Li-Po & Li-Po \\
Battery capacity & $2470 \mathrm{mAh}$ & $1900 \mathrm{mAh}$ & $3450 \mathrm{mAh}$ & $2700 \mathrm{mAh}$ \\
Removable battery & No & Yes & No & No \\
\hline
\end{tabular}

The experiment took place in a medical testing facility, in two separate rooms with independent climate controls. As previous work identified the effect of cold ambience on performance during mobile interaction [13], we replicated the protocol to determine if under the same conditions we could use battery temperature to infer finger temperature. Namely, we set the cold room temperature to $-10{ }^{\circ} \mathrm{C}$, with wind velocity below $0.1 \mathrm{~m} / \mathrm{s}$ and $70-75 \%$ humidity, while the warm room was set to $20^{\circ} \mathrm{C}$. All four smartphones were rotated between these two spaces to measure fluctuations in battery temperature: 14 minutes in cold temperature, 14 minutes in room temperature, 14 minutes in the cold temperature, and again 14 minutes in room temperature. We developed a custom Android application to record smartphone battery temperature for each device every 30 seconds during the experiment. Due to its power saving features, Android only allows polling of battery related data when an event is registered by the device's battery. Therefore, we ensured the triggering of an event on smartphones' battery every 30 seconds by plugging and unplugging the devices into an electrical outlet to collect battery temperature measurements.

3.1.1 Results. Fig. 1 shows the battery temperature fluctuation for each smartphone used in Experiment 1 . Vertical lines represent the start of the experiment in each of the rooms. We calculated the Pearson productmoment correlation coefficient between each device to assess the relationship between the measured battery temperatures. A statistically significant positive correlation was observed between the battery temperatures of all four devices (Table 2).

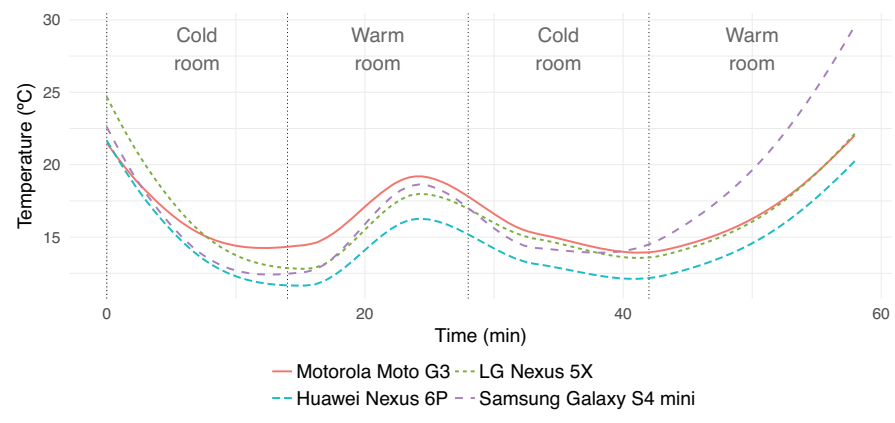

Fig. 1. Smartphone battery temperature change. 
Table 2. Pearson correlation coefficients between battery temperatures of the used smartphones.

\begin{tabular}{|c|c|c|c|c|c|}
\hline & & $\begin{array}{l}\text { Motorola } \\
\text { Moto G3 } \\
\end{array}$ & $\begin{array}{l}\text { Samsung } \\
\text { Galaxy S4 Mini }\end{array}$ & $\begin{array}{l}\text { Huawei } \\
\text { Nexus 6P }\end{array}$ & $\begin{array}{l}\text { LG } \\
\text { Nexus 5X }\end{array}$ \\
\hline Motorola Moto G3 & & - & 0.96 & 0.98 & 0.92 \\
\hline $\begin{array}{l}\text { Samsung Galaxy } \\
\text { Mini }\end{array}$ & S4 & 0.96 & - & 0.99 & 0.88 \\
\hline Huawei Nexus 6P & & 0.98 & 0.99 & - & 0.91 \\
\hline LG Nexus 5X & & 0.92 & 0.88 & 0.91 & - \\
\hline
\end{tabular}

\subsection{Experiment 2: Finger \& Battery Temperature}

In this experiment, we investigate if smartphone battery temperature and human finger temperature co-vary when exposed to changing ambient temperature. The experimental design was adopted from previous work [13]. We recruited 24 participants for our experiment through social media and mailing lists. We controlled for gender by having an equal distribution of males and females. All participants had lived in cold climates (e.g., Scandinavia) for more than six months and owned a smartphone for at least one year. We also controlled participants' clothing by instructing them to wear a single layer of trousers and top garments on the day of the study and providing them with additional winter attire.

After participants were briefed on the purpose of the study, we asked them to sign a consent form if they agreed to the study's specifications. Participants were then instrumented with thermal sensors attached below the nails of their index finger and thumb on the back of their dominant hand. Thermal data was logged every 1 second using a mobile battery-powered Grant Squirrel meter/logger series 1000 (Fig. 2), allowing us to measure finger temperature, as described in $[6,13]$. We chose to only instrument these two fingers as they are the ones most likely to be used during one-handed or two-handed interaction with a smartphone. Participants did not wear gloves during the experiment and were asked to not place their hands inside of their pockets. The experiment took place in the same medical facility and rooms as Experiment 1, using the same room temperatures. Participants were asked to wear additional winter attire provided by us when exposed to the cold chamber (Fig. 2). Following the design of Sarsenbayeva et al. [13], participants alternated between the two rooms (cold-warm-cold-warm), and we recorded both battery and finger temperature every 30 seconds. During the study, participants were asked to use a provided smartphone to complete target acquisition tasks for 4 minutes in 3 different instances (at 1:00, 6:00 and 11:00, with a 1-minute break in between) for each condition (cold/index, cold/thumb, warm/index, warm/thumb). The hand posture was randomised and counterbalanced. We avoided using colder temperatures in our study (i.e., below $-10^{\circ} \mathrm{C}$ ) to ensure the wellbeing of the participants. The experimental design was approved by the ethics committee of our university.

3.2.1 Results. A Pearson product-moment correlation coefficient was calculated to assess the relationship between participants' finger temperatures (index and thumb) and the phone's battery temperature. A positive correlation was observed for both fingers (index: $r=0.86, p<0.01$; thumb: $r=0.85, p<0.01$ ). Fig. 3 shows the changes in finger temperature and the smartphone's battery temperature throughout the experiment for each participant. Furthermore, our results show that participants' finger temperature decreased on average $0.73{ }^{\circ} \mathrm{C}$ per minute in the cold room, and increased on average $0.79^{\circ} \mathrm{C}$ per minute in the warm room. 

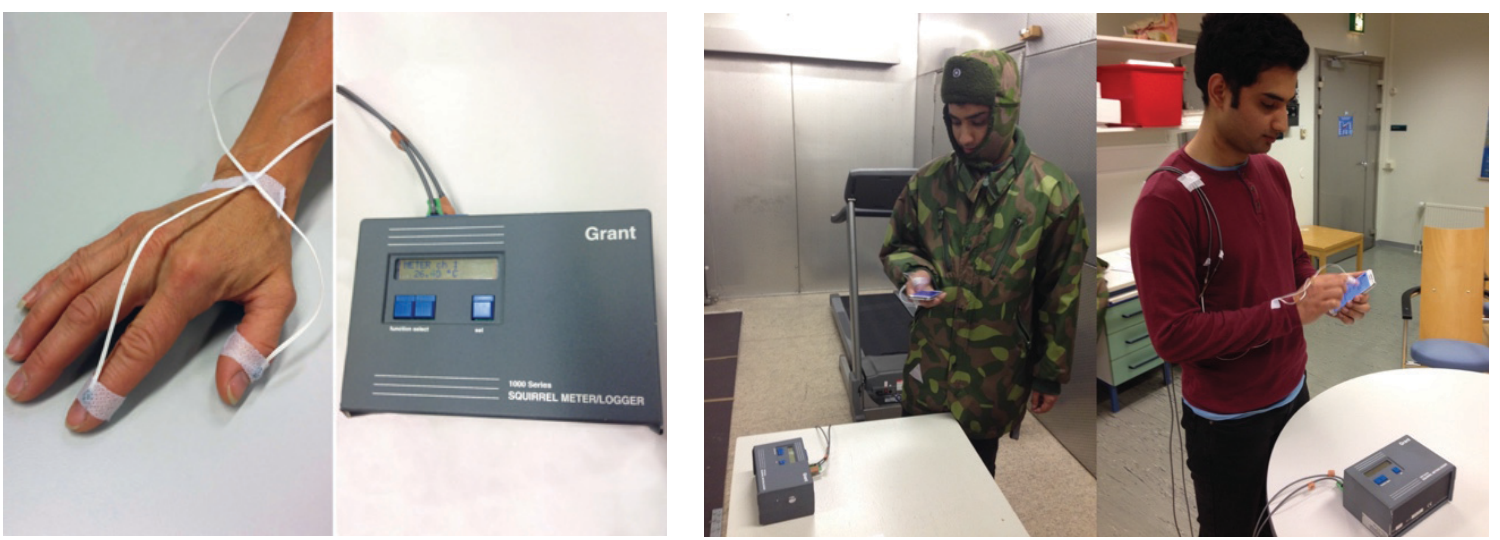

Fig. 2. Left: Grant Squirrel meter/logger (series 1000) and a participant's hand with thermal sensors attached. Right: Participant in the cold chamber and in the warm room.

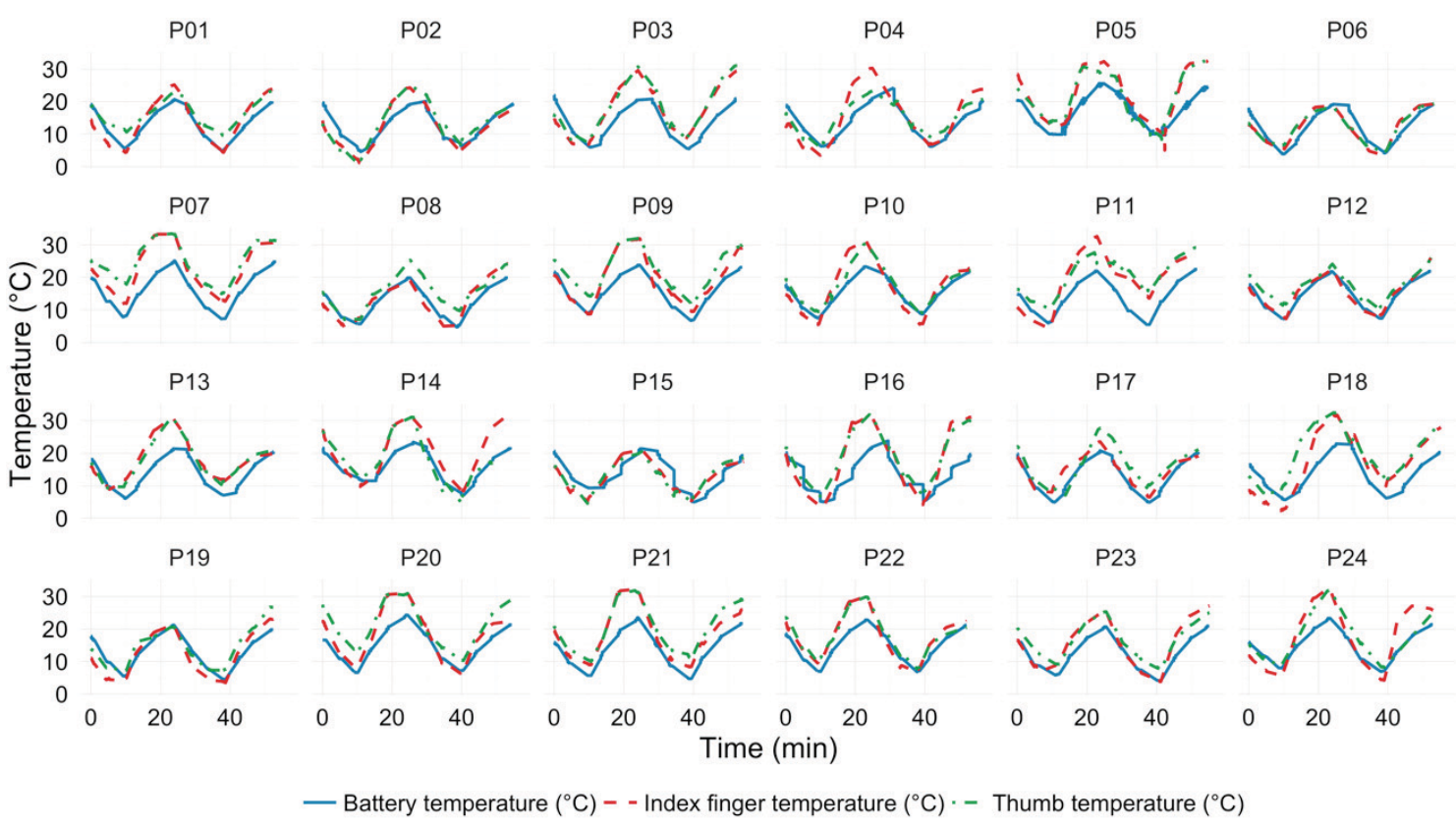

Fig. 3. Participants' finger temperature and smartphone battery temperature.

\section{DISCUSSION}

\subsection{Inferring Cold-Induced Situational Impairments}

Previous work has shown that ambient temperature is highly correlated with smartphone battery temperature $[1,12]$, and that ambient temperature has a significant impact on body temperature, particularly the extremities (e.g. fingers) [11]. In our work, we investigate the missing link regarding the relationship between finger temperature and smartphone battery temperature (Fig. 4). Our results demonstrate that smartphone battery 
temperature is highly correlated with users' finger temperature (index: $r=0.86$, thumb: $r=0.85$ ) and can therefore be used to infer potential cold-induced situational impairments during mobile interaction for both interaction modes (one-handed and two-handed).

Our work demonstrates that changes in smartphone battery temperature along with other factors (e.g., user input pattern) can be leveraged to signal the need to adapt the smartphone for cold environment. In addition, we show that this can be achieved without adding any additional sensors to the smartphone. Our approach of detecting a situational impairment corresponds to the method used by Goel et al. [4], where an accelerometer (a general-purpose sensor on most smartphones) was used to detect and reduce situational impairments induced by walking.

While previous work was able to achieve high accuracy when measuring device surface and screen temperature through additional sensors [3], an approach with add-on sensors aimed at measuring finger temperature is currently not feasible in real world situations. This is a challenge for researchers or interface designers who wish to adapt the interaction and interface when cold-induced situational impairments are detected. Our method shows that off-the-shelf smartphones can be used to detect users' finger temperature, thus providing a practical way to detect and accommodate for cold-induced situational impairments.

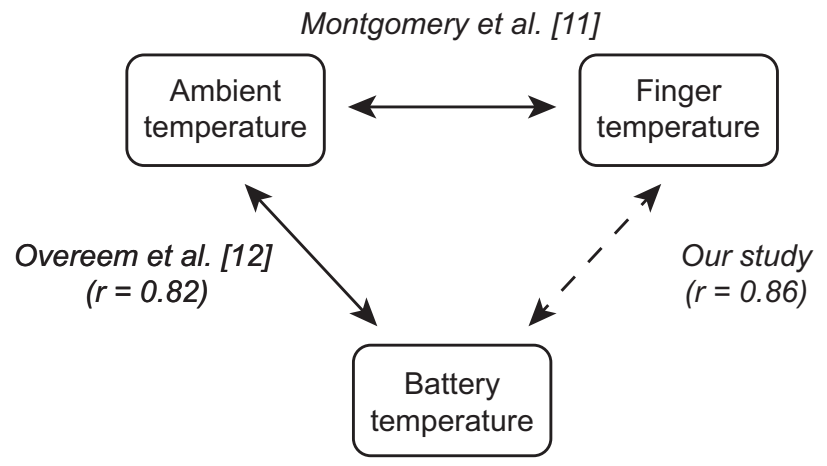

Fig. 4. Smartphone battery temperature relation to ambient temperature and finger temperature.

\subsection{Method Applicability}

Previous work has argued that cold environments cause significant deterioration of fine-motor performance when interacting with the mobile device $[6,13]$. To improve fine-motor performance during mobile interaction, it is important for the device to be aware of the changes in a user's finger temperature and adapt its interface accordingly. Here we present an unobtrusive method to detect these cold-induced situational impairments during mobile interaction.

In practice, our method can be used as follows. When a user initiates interaction with the device, the device registers its current battery temperature. Subsequent decreases in battery temperature while the user is still interacting with the phone can then be used to infer drops in finger temperature. Once a certain threshold of finger temperature decrease has occurred, the smartphone interface should then adapt accordingly or provide warnings to the user. It is important to note that for our method to work, finger temperature inference can only occur during interaction. When not using their phone, users might put their hands in their pockets or warm up their hands through other means.

Finally, previous work has shown that a decrease of just a few degrees Celsius can affect mobile interaction performance [6]. Here, we show that participants' finger temperature dropped on average $0.73{ }^{\circ} \mathrm{C}$ per minute under our cold exposure conditions. However, at temperatures lower than $-10^{\circ} \mathrm{C}$, the cooling rate of the fingers would be accelerated. This means that decreases in mobile interaction performance would occur in increasingly shorter spans of time. 


\subsection{Limitations}

This study has several limitations. While the choice for a constant room temperature is well motivated (temperature hardly changes during a typical session of smartphone usage [15]), we were limited in the actual temperature level imposed on our participants due to safety concerns. While outdoor temperatures in some parts of the world reach much lower temperatures than $-10^{\circ} \mathrm{C}$, we decided not to study more extreme scenarios. In addition, we have not been able to test a wider range of smartphone models due to both time and financial constraints. However, the range of smartphones used in our study (Table 1) does show a considerable level of diversity. This, combined with the high correlation in our results, provides us with sufficient confidence that our results hold true for a much larger selection of smartphone devices.

Further, participants were instructed to not wear gloves or warm their hand by any other means, unlike in naturalistic settings where they could wear touchscreen gloves to interact with their devices or warm their hands inside of their pockets. This was by design as we wanted to: 1) avoid touch inaccuracies during mobile interaction, and 2) observe steady finger temperature drop.

Finally, we did not run compute-intensive applications on the devices during our experiment as we chose to focus on more typical interactions that are more likely to occur in outdoor cold environments.

\section{CONCLUSION}

In this study, we demonstrate that changes in smartphone battery temperature can be used to infer changes in users' finger temperature. By doing so we also filled an important gap in the literature. While previous work has identified a relationship between ambient temperature and finger temperature, and between ambient temperature and battery temperature, our work is the first to establish a relationship between finger temperature and battery temperature. This is an important finding as it shows that cold-induced situational impairments can be predicted using off-the-shelf smartphones. This information can then be used to adapt mobile interfaces to overcome cold-induced situational impairments or simply to provide warnings to the user on over-exposure to cold environments.

\section{REFERENCES}

[1] S. Aram, A. Troiano and E. Pasero. 2012. Environment sensing using smartphone. IEEE Sensors Applications Symposium, 1-4. http://dx.doi.org/10.1109/SAS.2012.6166275.

[2] Trinh M. T. Do and Daniel Gatica-Perez. 2011. GroupUs: Smartphone Proximity Data and Human Interaction Type Mining. In Proceedings of the 2011 15th Annual International Symposium on Wearable Computers, IEEE Computer Society, 21-28. http://dx.doi.org/10.1109/ISWC.2011.28.

[3] Begum Egilmez, Gokhan Memik, Seda Ogrenci-Memik and Oguz Ergin. 2015. User-specific Skin Temperature-aware DVFS for Smartphones. In Proceedings of the 2015 Design, Automation \& Test in Europe Conference \& Exhibition, EDA Consortium, 1217-1220.

[4] Mayank Goel, Leah Findlater and Jacob Wobbrock. 2012. WalkType: using accelerometer data to accomodate situational impairments in mobile touch screen text entry. In Proceedings of the SIGCHI Conference on Human Factors in Computing Systems, 2687-2696. http://dx.doi.org/10.1145/2207676.2208662.

[5] Mayank Goel, Jacob Wobbrock and Shwetak Patel. 2012. GripSense: Using Built-in Sensors to Detect Hand Posture and Pressure on Commodity Mobile Phones. In Proceedings of the 25th Annual ACM Symposium on User Interface Software and Technology, ACM, 545-554. http://dx.doi.org/10.1145/2380116.2380184.

[6] Jorge Goncalves, Zhanna Sarsenbayeva, Niels van Berkel, Chu Luo, Simo Hosio, Sirkka Rissanen, Hannu Rintamäki and Vassilis Kostakos. 2016. Tapping Task Performance on Smartphones in Cold Temperature. Interacting with Computers, 29, 3: 355-367.

[7] G. Havenith, R. Heus and H. A. Daanen. 1995. The hand in the cold, performance and risk. Arctic Med Res 54 Suppl 2: 37-47.

[8] Richard Honicky, Eric A. Brewer, Eric Paulos and Richard White. 2008. N-smarts: Networked Suite of Mobile Atmospheric Realtime Sensors. In Proceedings of the Second ACM SIGCOMM Workshop on Networked Systems for Developing Regions, ACM, 25-30. http://dx.doi.org/10.1145/1397705.1397713.

[9] Weiwei Jiang, Denzil Ferreira, Jani Ylioja, Jorge Goncalves and Vassilis Kostakos. 2014. Pulse: Low bitrate wireless magnetic communication for smartphones. In International foint Conference on Pervasive and Ubiquitous Computing, ACM, 261-265. http://dx.doi.org/10.1145/2632048.2632094.

[10] Clifford F. Mass and Luke E. Madaus. 2014. Surface Pressure Observations from Smartphones: A Potential Revolution for HighResolution Weather Prediction? Bulletin of the American Meteorological Society 95, 9: 1343-1349. http://dx.doi.org/10.1175/BAMS-D13-00188.1.

[11] Leslie D. Montgomery and Bill A. Williams. 1976. Effect of Ambient Temperature on the Thermal Profile of the Human Forearm, Hand, and Fingers. Annals of Biomedical Engineering 4, 3: 209-219. http://dx.doi.org/10.1007/BF02584515. 
[12] Aart Overeem, J. Robinson, Hidde Leijnse, Gert-Jan Steeneveld, B. P Horn and Remko Uijlenhoet. 2013. Crowdsourcing urban air temperatures from smartphone battery temperatures. Geophysical Research Letters 40, 15: 4081-4085.

[13] Zhanna Sarsenbayeva, Jorge Goncalves, Juan García, Simon Klakegg, Sirkka Rissanen, Hannu Rintamäki, Jari Hannu and Vassilis Kostakos. 2016. Situational Impairments to Mobile Interaction in Cold Environments. In International foint Conference on Pervasive and Ubiquitous Computing, ACM 85-96. http://dx.doi.org/10.1145/2971648.2971734.

[14] Frans Snik, Jeroen H. Rietjens, Arnoud Apituley, Hester Volten, Bas Mijling, Antonio Di Noia, Stephanie Heikamp, Ritse C. Heinsbroek, Otto P. Hasekamp, J. M. Smit, Jan Vonk, Daphne M. Stam, Gerard van Harten, Jozua de Boer and Christoph U. Keller. 2014. Mapping atmospheric aerosols with a citizen science network of smartphone spectropolarimeters. Geophysical Research Letters 41, 20: 7351-7358. http://dx.doi.org/10.1002/2014GL061462.

[15] Niels van Berkel, Chu Luo, Theodoros Anagnostopoulos, Denzil Ferreira, Jorge Goncalves, Simo Hosio and Vassilis Kostakos. 2016. A Systematic Assessment of Smartphone Usage Gaps. In Conference on Human Factors in Computing Systems, ACM, 4711-4721. http://dx.doi.org/10.1145/2858036.2858348.

[16] Jacob O. Wobbrock. 2006. The future of mobile device research in HCI. In CHI 2006 workshop proceedings: what is the next generation of human-computer interaction, 131-134. 